TITLE:

\title{
Magnetization process analysis using a simplified domain structure model
}

\author{
$\operatorname{AUTHOR}(\mathrm{S})$ :
}

Matsuo, T.

\section{CITATION:}

Matsuo, T.. Magnetization process analysis using a simplified domain structure model. JOURNAL OF APPLIED PHYSICS 2011, 109(7): $07 D 332$.

\section{ISSUE DATE:}

2011-04

URL:

http://hdl.handle.net/2433/160649

\section{RIGHT:}

Copyright 2011 American Institute of Physics. This article may be downloaded for personal use only. Any other use requires prior permission of the author and the American Institute of Physics. The following article appeared in JOURNAL OF APPLIED PHYSICS 109, 07D332 (2011) and may be found at http://link.aip.org/link/?jap/109/07D332 


\section{AIP $\mid$ Applied Physics}

Magnetization process analysis using a simplified domain structure model

T. Matsuo

Citation: J. Appl. Phys. 109, 07D332 (2011); doi: 10.1063/1.3556923

View online: http://dx.doi.org/10.1063/1.3556923

View Table of Contents: http://jap.aip.org/resource/1/JAPIAU/v109/i7

Published by the American Institute of Physics.

\section{Related Articles}

Current-induced synchronized switching of magnetization

Appl. Phys. Lett. 101, 062408 (2012)

Modeling and simulation of high voltage and radio-frequency transformer

J. Appl. Phys. 111, 07E737 (2012)

Enhancement of perpendicular magnetic anisotropy in FeB free layers using a thin $\mathrm{MgO}$ cap layer J. Appl. Phys. 111, 07 C723 (2012)

Effect of enhanced damping caused by spin-motive force on vortex dynamics J. Appl. Phys. 111, 07D120 (2012)

Extended frequency analysis of magnetic losses under rotating induction in soft magnetic composites J. Appl. Phys. 111, 07E325 (2012)

\section{Additional information on J. Appl. Phys.}

Journal Homepage: http://jap.aip.org/

Journal Information: http://jap.aip.org/about/about_the_journal

Top downloads: http://jap.aip.org/features/most_downloaded

Information for Authors: http://jap.aip.org/authors

\section{ADVERTISEMENT}

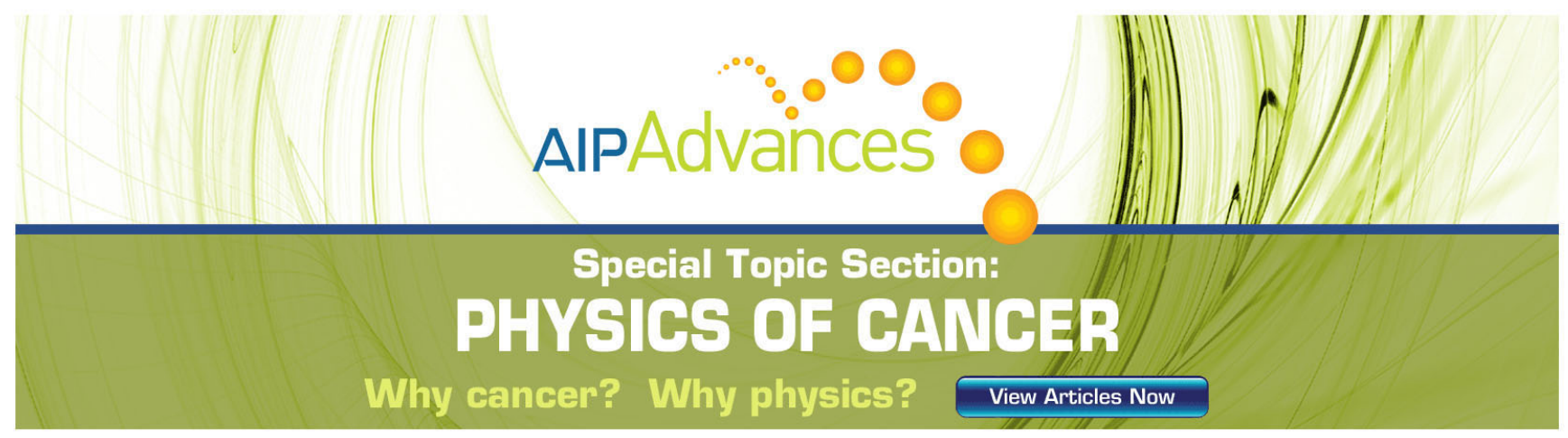




\title{
Magnetization process analysis using a simplified domain structure model
}

\author{
T. Matsuo ${ }^{1, a)}$ \\ Graduate School of Engineering, Kyoto University, Kyoto 615-8510, Japan
}

(Presented 15 November 2010; received 23 September 2010; accepted 27 November 2010; published online 31 March 2011)

\begin{abstract}
A domain structure model (DSM) is simplified to analytically reveal the basic mechanism of the mesoscopic magnetization process. Magnetic energy components are normalized by the anisotropic energy to compare the influence of individual energy components explicitly. The magnetostatic field is approximated as the product of averaged magnetization and demagnetizing factors. A simple two-domain model represents the domain wall motion, nucleation-type hysteresis, and magnetization rotation. Magnetization curves obtained by the simplified DSM agree with those given by the periodic micromagnetic simulation. The simplified DSM represents the discontinuous magnetization-state transition observed in a magneto-impedance sensor. (C) 2011 American Institute of Physics. [doi:10.1063/1.3556923]
\end{abstract}

\section{INTRODUCTION}

The micromagnetic simulation (MMS) solving the LLG equation $^{1,2}$ is a powerful tool for describing microscopic behavior of magnetic materials. However, it is difficult for the micromagnetic simulation to analyze mesoscopic ( $\mu \mathrm{m}$ - to mm-length scales) or macroscopic magnetization, because of high computational costs. Several domain structure models (DSMs; Refs. 3-5) have been proposed to represent the mesoscopic behavior of magnetic materials and have been successfully applied to the analysis of magnetic thin films. The comparison of a DSM with a micromagnetic simulation ${ }^{6}$ showed that the DSM can represent magnetization processes efficiently.

Despite the computational efficiency of the DSM, the energy computation and minimization procedures of the DSM are not always simple. Therefore, it is sometimes difficult to understand the mechanism behind the magnetization process obtained by the DSM. For the DSM to be a useful tool for development of magnetic devices, such as the magneto-impedance (MI) sensor, ${ }^{7}$ the physical meanings of the model parameters should be sufficiently clear to explain the magnetic properties simulated by the DSM.

This study simplifies the DSM to analytically reveal the basic mechanisms of mesoscopic magnetization process including domain wall motion, which is not represented by single-domain models. Magnetic energy components are normalized to compare the influence of individual energy components directly using the model parameters.

\section{DOMAIN STRUCTURE MODEL AND ITS SIMPLIFICATION}

The domain structure of the DSM is given by several domains with uniform magnetization vectors and their boundaries (domain walls). Two-dimensional domain structures are studied in this paper to analyze thin magnetic materials.

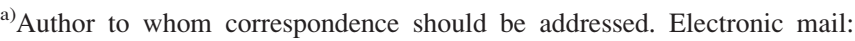
tmatsuo@kuee.kyoto-u.ac.jp.
}

Figure 1(a) provides an example of the domain structure where $\boldsymbol{m}_{1}, \ldots, \boldsymbol{m}_{4}$ are normalized magnetization vectors. The directions of the magnetization vectors and the locations of domain walls are determined to minimize the total magnetic energy $E ; E$ is given by the summation of the anisotropic energy $E_{\text {an }}$, the Zeeman energy $E_{\text {ap }}$, the domain wall energy $E_{w}$, and the magnetostatic energy $E_{\mathrm{st}}$.

The anisotropic energy is given as

$$
E_{\mathrm{an}}=K_{1} d \sum_{i=1}^{N \mathrm{~d}} S_{i} \sin ^{2}\left(\theta_{i}-\theta_{e}\right),
$$

where uniaxial anisotropy is assumed; $K_{1}$ is the anisotropy constant, $N_{\mathrm{d}}$ is the number of domains, $\theta_{e}$ is the direction of the easy axis, $d$ is the thickness of the material, and $S_{i}$ is the area of the $i$-th magnetic domain. In this study, the energy components are normalized as

$$
\begin{gathered}
e_{\mathrm{an}}=E_{\mathrm{an}} /\left(V K_{1}\right)=\sum_{i=1}^{N \mathrm{~d}} \lambda_{i} \sin ^{2}\left(\theta_{i}-\theta_{e}\right), \\
V=d \sum_{i=1}^{N \mathrm{~d}} S_{i}, \lambda_{i}=S_{i} d / V .
\end{gathered}
$$

The Zeeman energy is given by

$$
\begin{gathered}
e_{\mathrm{ap}}=E_{\mathrm{ap}} /\left(V K_{1}\right)=-2 h \sum_{i=1}^{N \mathrm{~d}} \lambda_{i} \cos \left(\theta_{i}-\theta_{\mathrm{H}}\right), \\
h=H_{\mathrm{ap}} / \kappa M_{\mathrm{s}}, \kappa=2 K_{1} / \mu_{0} M_{\mathrm{s}}^{2},
\end{gathered}
$$

where $\left(M_{\mathrm{s}}, \theta_{i}\right)$ are the magnitude and rotation angles of spontaneous magnetization, respectively, and $\left(H_{\mathrm{ap}}, \theta_{\mathrm{H}}\right)$ are those of the applied magnetic field.

A simple Bloch wall model gives the domain wall energy ${ }^{4}$ as

$$
e_{\mathrm{w}}=E_{\mathrm{w}} /\left(V K_{1}\right)=w \sum_{j=1}^{N \mathrm{w}}\left(L_{j} / L\right)\left(1-\cos \theta_{\mathrm{w} j}\right) / 2
$$



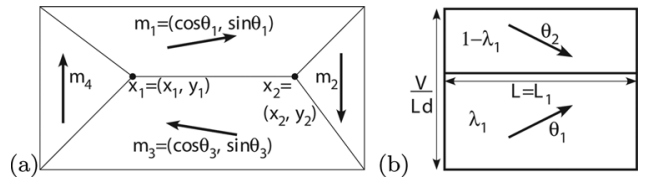

FIG. 1. Domain structure model: (a) domains and domain walls and (b) the two-domain model.

$$
w=4 l_{\mathrm{K}} L d / V, l_{\mathrm{K}}=\sqrt{A / K_{1}},
$$

where a wall energy per unit area of $4 \sqrt{A K_{1}}$ is assumed; $N_{\text {w }}$ is the number of domain walls, $A$ is the exchange stiffness constant, $L_{j}$ is the length of the $j$-th domain wall, $L$ is a typical domain wall length, $l_{\mathrm{K}}$ is an exchange length, ${ }^{2}$ and $\theta_{\mathrm{w} j}$ is the difference angle of the adjacent magnetization vectors of the $j$-th domain wall.

The magnetostatic energy $E_{\mathrm{st}}$ is the most complex component to compute. This study highly simplifies the magnetostatic energy computation, and the magnetostatic field, $\boldsymbol{H}_{\mathrm{st}}$, is assumed to be proportional to an average magnetization uniformly as

$$
\boldsymbol{H}_{\mathrm{st}}=-M_{\mathrm{s}}\left(k_{x} \sum_{i=1}^{N \mathrm{~d}} \lambda_{i} \cos \theta_{i}, k_{y} \sum_{i=1}^{N \mathrm{~d}} \lambda_{i} \sin \theta_{i}\right),
$$

where $\left(k_{x}, k_{y}\right)$ are the demagnetizing factors, which are assumed to be constant. Thereby, the normalized magnetostatic energy is given by

$$
\begin{gathered}
e_{\mathrm{st}}=E_{\mathrm{st}} /\left(V K_{1}\right)=\sum_{i=1}^{N \mathrm{~d}} \sum_{j=1}^{N \mathrm{~d}} \lambda_{i} \lambda_{j}\left(s_{x} \cos \theta_{i} \cos \theta_{j}\right. \\
\left.+s_{y} \sin \theta_{i} \sin \theta_{j}\right), \\
s_{x}=k_{x} / \kappa, s_{y}=k_{y} / \kappa .
\end{gathered}
$$

The effect of material or domain shape becomes abstracted by the approximation of $e_{\mathrm{st}}$. For example, the material size affects only $L_{j}$. This rather rough approximation [Eq. (8)] can be justified by assuming a periodic alignment of same-domain structures, ${ }^{8}$ because the magnetostatic effect from large distances depends primarily on the average magnetization.

The parameter $h$ represents the magnitude of applied magnetic field. The parameters $\left(s_{x}, s_{y}\right)$ represent the influence of magnetostatic field, whereas $w$ represents the influence of energy cost to have domain walls. Because of the global approximation of magnetostatic field, the local nonuniformity of magnetostatic field due to the domain structure is not taken into account. The nonuniformity causes an additional energy cost that is not given by $e_{\mathrm{st}}$ and $e_{\mathrm{w}}$ in Eq. (7). This paper simply includes all the energy costs to have the domain wall at the domain wall energy adjusting $w$, which has a minimum value given by Eq. (7).

When $w=0$, the simplified DSM becomes equivalent to phase theory, ${ }^{1}$ because of the simplification of the magnetostatic field computation. When $w \rightarrow \infty$, the simplified DSM describes the behavior of the Stoner-Wohlfarth particle, ${ }^{1,2}$ because all the magnetization directions coincide.

For simplicity, the number of domains is limited to two with $L=L_{1}$ as shown in Fig. 1(b) in this paper. The magnet- ization is determined by finding $\boldsymbol{X}=\left(\theta_{1}, \theta_{2}, \lambda_{1}\right)$ such that it locally minimizes $e=E /\left(V K_{1}\right)$. The total energy becomes a local extremum when

$$
\partial e / \partial \boldsymbol{X}=0 .
$$

Its solution gives a local minimum for $e$ when all the eigenvalues of $\partial^{2} e / \partial \boldsymbol{X}^{2}$ are positive.

\section{MAGNETIZATION PROCESS ANALYSIS}

\section{A. Magnetization curves}

The variation of parameter $h$ with fixed $\theta_{\mathrm{H}}$ means the application of an alternating magnetic field. Figure 2 shows magnetization curves numerically obtained with $\theta_{e}=0$, $\theta_{\mathrm{H}}=0$, and $\left(w, s_{x}, s_{y}\right)=(4,0.5,0.5),(0.5,4,4),(0.5,2,6)$, $(0.56,2)$ where normalized average magnetization $m_{x}=\lambda_{1} \cos \theta_{1}+\left(1-\lambda_{1}\right) \cos \theta_{2}$ is plotted. The solid lines indicate the stable solutions that give local energy minima. The relation $s_{x}<s_{y}$ (or $s_{x}>s_{y}$ ) implies a shape anisotropy having the easy direction along the $x$ - (or $y$-) direction. When $\theta_{\mathrm{H}}=0$, the stable solutions are classified into three types: [S] the single domain type where $\theta_{1}=\theta_{2}$ and $\lambda_{1}=1 / 2$, [WM] the $180^{\circ}$ domain-wall motion type where $\theta_{1}=0$ and $\theta_{2}=\pi$ or vice versa, and [R] the magnetization rotation type with $\theta_{2}=-\theta_{1}$ and $\lambda_{1}=1 / 2$. Stable solutions in Fig. 2 have labels "S," "WM," and "R" corresponding to the classifications given above. Due to the WM- and R-type solutions, the magnetization properties of two-domain model substantially differ from that of single-domain model represented by the Stoner-Wohlfarth particle.

When $\theta_{\mathrm{H}}=0$, Eq. (11) has the S-type solution of $\boldsymbol{X}$ $=(0,0,1 / 2)$, which is stable when $h>h_{S}=\max \left(s_{x}-w-1\right.$, $\left.s_{x}-s_{y}-1\right)$. The circles in Fig. 2 indicate the points of $\pm\left(h_{S}, 1\right)$.

When $\theta_{\mathrm{H}}=0$, Eq. (11) also has the WM-type solution of $\boldsymbol{X}=\left(0, \pi, h / 2 s_{x}+1 / 2\right)$, which is stable when $|h|<h_{W M}$ $=s_{x} \sqrt{\left(1+s_{y}-w\right) /\left(1+s_{y}+s_{y} w\right)}$. When $s_{y}<w-1$, the WM-type solution is not stable. When $s_{y}>w-1$, the linear magnetization $m_{x}=h / s_{x}$ caused by the WM-type solution is seen in the range $|h|<h_{W M}$ as shown in Figs. 2(b)-2(d). The squares in Fig. 2 indicate the points where $\left(h, m_{x}\right)$
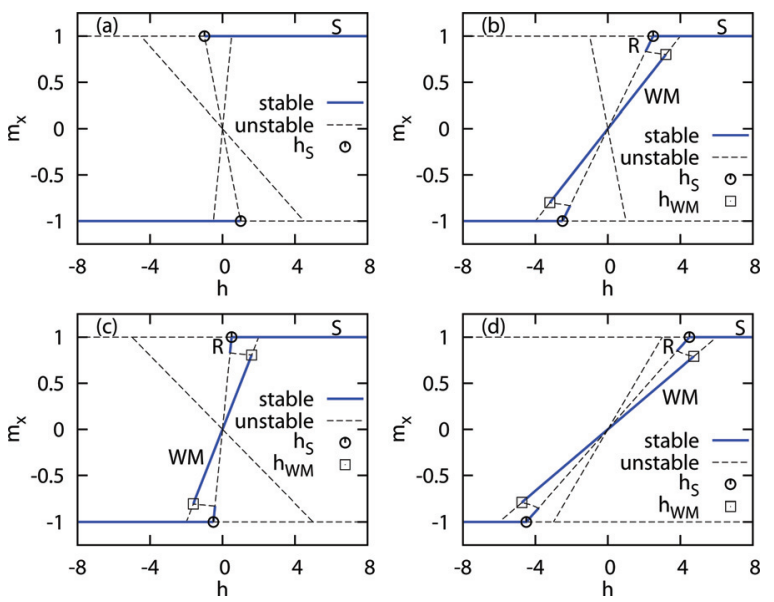

FIG. 2. (Color online) Magnetization properties with $\theta_{H}=\theta_{e}=0$ : (a) $\left(w, s_{x}, s_{y}\right)=(4,0.5,0.5),(\mathrm{b})(0.5,4,4),(\mathrm{c})(0.5,2,6)$, and (d) $(0.5,6,2)$. 
$= \pm\left(h_{W M}, h_{W M} / s_{x}\right)$. The WM-type solution appears to minimize the magnetostatic energy when $\left(s_{x}, s_{y}\right)$ are large and $w$ is small.

When $w$ is large and $\left(s_{x}, s_{y}\right)$ are small, the WM-type solution is suppressed and the S-type solution becomes dominant as shown in Fig. 2(a). This is because the domain wall energy is at a minimum when $\theta_{2}=\theta_{1}$ and at a maximum when $\theta_{2}=\theta_{1}+\pi$. Consequently, nucleation-type hysteresis appears.

When $\theta_{\mathrm{H}}=0$, the R-type solution of $\boldsymbol{X}=\left(\theta_{1},-\theta_{1}, 1 / 2\right)$ yields a magnetization that is proportional to $h$ as $m_{x}=h /\left(s_{x}-w-1\right)$. The transition from S-type to R-type reduces the magnetostatic energy without increasing the demagnetizing field along the $y$-direction.

\section{B. Comparison with micromagnetic simulation}

A magnetic material with uniaxial anisotropy was analyzed using the periodic MMS (Ref. 8) with $K_{1}=1 \times 10^{3} \mathrm{~J} / \mathrm{m}^{3}$, $A=1.3 \times 10^{-11} \mathrm{~J} / \mathrm{m}$, and $\mu_{0} M_{s}=1 \mathrm{~T}$. The periodic structure with a spatial period of $L_{x} \times L_{y} \times L_{z}=1.28 \times 0.64 \times 0.04$ $\mu \mathrm{m}^{3}$ is assumed. The magnetostatic field is computed from the magnetization distribution within the virtually enlarged computational domain, $\Omega_{v}=2 p_{x} L_{x} \times 2 p_{y} L_{y} \times 2 p_{z} L_{z}$, in the periodic MMS, in which $\left(p_{x}, p_{y}, p_{z}\right)$ are called truncation periods.

The demagnetizing factors, $\left(k_{x}, k_{y}\right)$, in Eq. (8) are given by the macroscopic demagnetizing factors, ${ }^{8}$ which are geometrically determined from $\Omega_{v}$. The truncation periods $\left(p_{x}, p_{y}, p_{z}\right)$ are set at $(5,10,160),(5,10,80),(80,80,10)$, and $(80,40,10)$, which correspond to $\left(s_{x}, s_{y}\right)$ of $(133,133)$, $(82,82),(0.45,1.77)$, and $(0.24,3.84)$, respectively. Equation (7) yields a value of 1.4 for $w$, because two-domain walls exist in the spatial period. A priori or exact evaluation of the additional energy cost to have the domain walls is not easy. It is roughly assumed to balance with the wall energy using Eq. (7). Thus, $w$ is set at 2.8 .

Figure 3(a) depicts MH curves in the magnetostatically isotropic cases: $s_{x}=s_{y}=133,82$, and Fig. 3(b) depicts the anisotropic cases: $\left(s_{x}, s_{y}\right)=(0.45,1.77)$ and $(0.24,3.84)$. The simplified DSM gives the approximate magnetization properties with negligible computational time compared with that of periodic MMS.

\section{Analysis of magneto-impedance sensor}

A thin film MI sensor ${ }^{7,9}$ is analyzed, where the steplike MI change is caused by the discontinuous magnetizationstate transition between the single and stripe domains. An amorphous $\mathrm{Co}_{85} \mathrm{Nb}_{12} \mathrm{Zr}_{3}$ film of $2000 \times 20 \times 2 \mu \mathrm{m}^{3}$ has parameters $A=1.49 \times 10^{-11} \mathrm{~J} / \mathrm{m}$ and $\mu_{0} M_{s}=0.93 \mathrm{~T}$. The slanted
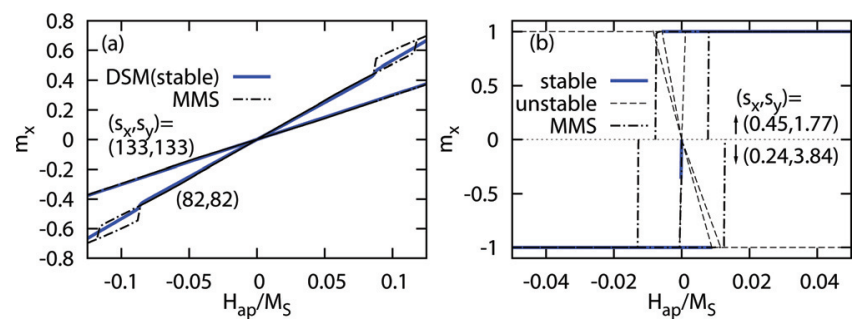

FIG. 3. (Color online) Comparison with the periodic MMS: (a) $s_{x}=s_{y}$ $=133,82$, and $(\mathrm{b})\left(s_{x}, s_{y}\right)=(0.45,1.77),(0.24,3.84)$.
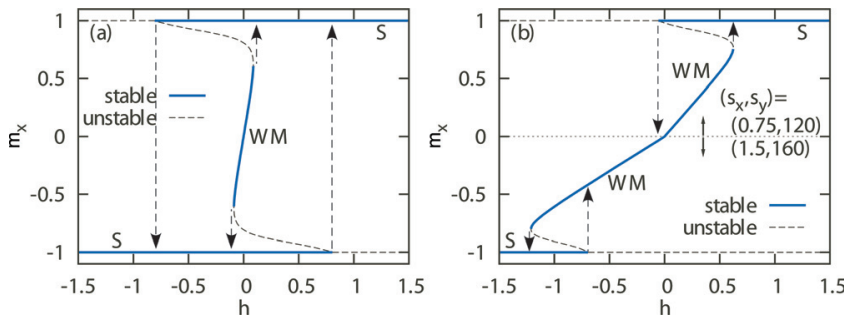

FIG. 4. (Color online) Magnetization-state transition of the MI sensor: (a) $\left(s_{x}, s_{y}\right)=(0.0,80)$, and (b) $(0.75,120),(1.5,160)$.

stripe domains are developed through the induced anisotropy with $\theta_{e}=30^{\circ}$ and $K_{1}=260 \mathrm{~J} / \mathrm{m}^{3}$. The single domain has a magnetization along the longitudinal direction, which is assigned to the $x$-direction.

The parameters $\left(s_{x}, s_{y}\right)$ are determined by the magnetostatic field, $\boldsymbol{H}_{\mathrm{st}}$, yielded by the uniform magnetization along the $x$ - and $y$-directions. However, $\boldsymbol{H}_{\mathrm{st}}$ is not uniform along the $x$-direction because of its long shape. The averaged $\boldsymbol{H}_{\mathrm{st}}$ gives $\left(s_{x}, s_{y}\right) \simeq(1.5,160)$, whereas $\boldsymbol{H}_{\text {st }}$ at the film center gives $(0.0,80)$. The parameter $w$ is set at 0.3 , which includes the energy cost to have the slanted closure domain. ${ }^{7}$

The MH curves are plotted in Fig. 4 with $\left(s_{x}, s_{y}\right)=(0.0$, $80),(0.75,120),(1.5,160)$, where the WM-type solution gives the stripe domains. The discontinuous magnetizationstate transition is represented in Fig. 4(b), which cannot be obtained using the phase theory $(w=0)$. In spite of the nonuniform demagnetizing field, the DSM achieves qualitative agreement with measured properties. ${ }^{9}$ The WM-type solution results in the linear magnetization represented by $m_{x}=h /$ $\left[s_{x}+(1-w) \sin ^{2} \theta_{e}\right]$ whereas the S-type solution is stable when $h>s_{x}-w-\cos \left(2 \theta_{e}\right)$ under the approximation $m_{y} \simeq 0$, because of a large $s_{y}$. The susceptibility of the WM-type solution roughly agrees with the measured value when $s_{x}=0$, because the stripe domains are observed around the center part of the film. The transition point from the S-type to the WMtype solution roughly agrees with the measured value when $s_{x}=0.75$. A four-domain or higher multi-domain model is required to obtain the qualitative agreement without an inconsistent parameter set, which will be addressed in future work.

\section{ACKNOWLEDGMENTS}

The author would like to express his gratitude to Dr. T. Nakai, Industrial Technology Institute, Miyagi Prefectural Government, and Professor K. Ishiyama, Tohoku University, for providing the MI-sensor data and for useful discussions.

${ }^{1}$ A. Hubert and R. Schäfer, Magnetic Domains: The Analysis of Magnetic Microstructures (Springer-Verlag, Berlin Heidelberg, 1998).

${ }^{2}$ H. Kronmüller and M. Fähnle, Micromagnetism and the Microstructure of Ferromagnetic Solids (Cambridge University Press, Cambridge, 2003).

${ }^{3}$ N. Smith, IEEE Trans. Magn. 24, 2380 (1988).

${ }^{4}$ C. Saka, K. Shiiki, and K. Shinagawa, J. Appl. Phys. 68, 263 (1990).

${ }^{5}$ M. Enokizono, T. Todaka, and Y. Midou, IEEE Trans. Magn. 32, 1172 (1996).

${ }^{6}$ T. Matsuo, N. Mimuro, and M. Shimasaki, J. Magn. Magn. Mater. 320, e1029 (2008).

${ }^{7}$ T. Nakai, K. Ishiyama, and J. Yamasaki, J. Appl. Phys. 101, 09N106 (2007).

${ }^{8}$ T. Matsuo and Y. Yamazaki, "Demagnetizing field in micromagnetic simulation under periodic boundary condition," IEEE Trans. Magn. (to be published).

${ }^{9}$ T. Nakai, K. Takada, and K. Ishiyama, IEEE Trans. Magn. 45, 3499 (2009). 